\title{
Unilateral tonsillar swelling: role and urgency of tonsillectomy
}

\begin{abstract}
Unilateral tonsillar swelling is a fairly common presenting complaint in an Ear, Nose and Throat (ENT) department. It may or may not be associated with any other symptoms. Most of the time, the tonsil asymmetry is secondary to previous history of tonsillitis, quinsy, and tonsil stones. Other benign lesions to cause tonsil swelling may include a mucus retention cyst, lipoma, polyp or papilloma. Sometimes, it is the site of primary malignancy but in these situations, it is often associated with red flag symptoms like pain in the mouth, dysphagia, odynophagia, referred otalgia, weight loss, night sweating, haemoptysis, haematemesis, hoarseness or neck nodes. Most of the patients with suspected tonsillar malignancy have underlying risk factors like smoking and excessive alcohol intake. However, lately, the tonsil squamous cell carcinoma can be found in younger patients with no history of smoking or drinking as there is rising incidence of human papilloma virus related oropharyngeal malignancy. Sometimes, lymphoma may manifest as a tonsil enlargement. If, after detailed history and examination, there remains any doubt about the underlying cause of unilateral tonsil swelling then tonsillectomy should be considered for histological analysis. it is our practice to perform bilateral tonsillectomy in such cases. We present a case of unilateral tonsillar swelling and discuss its management.
\end{abstract}

Volume | 3 Issue | - 202|

\section{J Kynaston, S Drever, M Shakeel, M Supriya, N McCluney}

Department of otolaryngology and head \&neck surgery, Aberdeen Royal Infirmary, Aberdeen, UK

Correspondence: Muhammad Shakeel, FRCSED (ORLHNS), Consultant ENT/Thyroid surgeon, Department of Otolaryngology-Head and Neck surgery, Aberdeen Royal Infirmary, Aberdeen, AB252ZN, UK, Tel 0044I 2245521 I7, Email drshakeel@doctors.org.uk

Received: June 24, 2020 | Published: February 25, 2021

Keywords: unilateral, tonsils, swelling, lymphoma, cancer, tonsillectomy, histology

\section{Introduction}

Unilateral tonsillar swelling is a fairly common presenting complaint in an Ear, Nose and Throat (ENT) department. It may or may not be associated with any other symptoms. Most of the time, the tonsil asymmetry is secondary to previous history of tonsillitis, quinsy, and tonsil stones. Other benign lesions to cause tonsil swelling may include a mucus retention cyst, lipoma, polyp or papilloma. Sometimes, it is the site of primary malignancy. Malignancy of the tonsils is a rare diagnosis and can present with few symptoms and signs. Thus, a high degree of clinical suspicion is warranted when assessing such a patient. Asymmetrical tonsillar swelling is a common reason for referral to the otolaryngologists to rule out malignancy.

\section{Aims}

To share management of a patient presenting with a left irregular tonsillar swelling masquerading as a malignancy. We discuss the presenting symptoms, examination findings, investigations and management.

\section{Case report}

A 46 year old man was urgently referred by his general practitioner (GP) to the head and neck clinic with two weeks history of a left sided sore throat and left sided unilateral tonsillar swelling. He was initially treated with antibiotics with no improvement; hence he was referred as a 'suspected cancer'. This tee-total, non-smoking man presented to the clinic with a persistent left sided sore throat with intermittent odynophagia. Otherwise, he was well with no significant past medical history. On examination, his oral cavity was normal. Examination of the oropharynx revealed a growth on the patients left tonsil. It was noted to be a cauliflower like polypoidal lesion measuring roughly 2 to $3 \mathrm{~cm}$. It was also noted that a small lymph node was palpable in left posterior triangle of the patient's neck. At this point a carcinoma or lymphoma was suspected but the clinical picture was not diagnostic. The routine blood tests were all normal with no evidence any acute infection. As per our departmental protocol, a biopsy was arranged to decide about the most appropriate imaging modality. In some institutions carrying out a cross sectional imaging like CT scan is allowed and is perfectly reasonable option to use. We, however, could not use this option because of our departmental protocol where imaging modality is guided by strong clinical judgement, cytology or an initial biopsy result. A tonsillar biopsy was taken in the clinic under local anaesthetic. The results showed focal scarring of subepithelial tissue with no evidence of dysplasia or malignancy. As the patient remained symptomatic and the clinical appearance of the enlarged tonsil was not entirely satisfactory, he was consented for a tonsillectomy in order to obtain a larger sample. The second histology report showed squamous mucosa with underlying tonsillar tissue and extensive areas of dense scarring which may account for its irregular macroscopic presentation. There was no evidence of malignancy. In the light of this reassuring histology no further investigations or treatment were undertaken. The patient made a complete recovery with resolution of his throat discomfort. The patient was discharged back to his GP.

\section{Discussion and conclusion}

Malignancy of the tonsils has an annual incidence of $0.5 \%$ of all new malignancies in the US and thus represents a small number of head and neck malignancies. The most common and important two types of tonsillar malignancy are squamous cell carcinoma and lymphoma. Treatment depends on staging but usually involves surgical resection followed by radiotherapy. Mortality again varies with staging but can be as high as $80 \%$ within 5 years of treatment. ${ }^{1}$ It is the possibility of tonsillar malignancy that alerts us when confronted with a unilateral tonsillar enlargement. It is quite often seen in outpatient clinics and can commonly present with relatively few signs and symptoms. This was true of our case. In such cases 
the choice and intensity of investigation can prove challenging. It has been demonstrated that asymmetrical tonsillar swelling (Figure 1) is a significant sign when diagnosing tonsillar malignancy. ${ }^{2}$ In such cases the next step is to perform further histological investigations to rule out a possible malignancy. This commonly involves an elective tonsillectomy. The cross sectional imaging like CT scan or MRI scan can be used to investigate suspected tonsillar malignancy. However, blood tests are not useful in such scenarios but can help rule out acute infection.

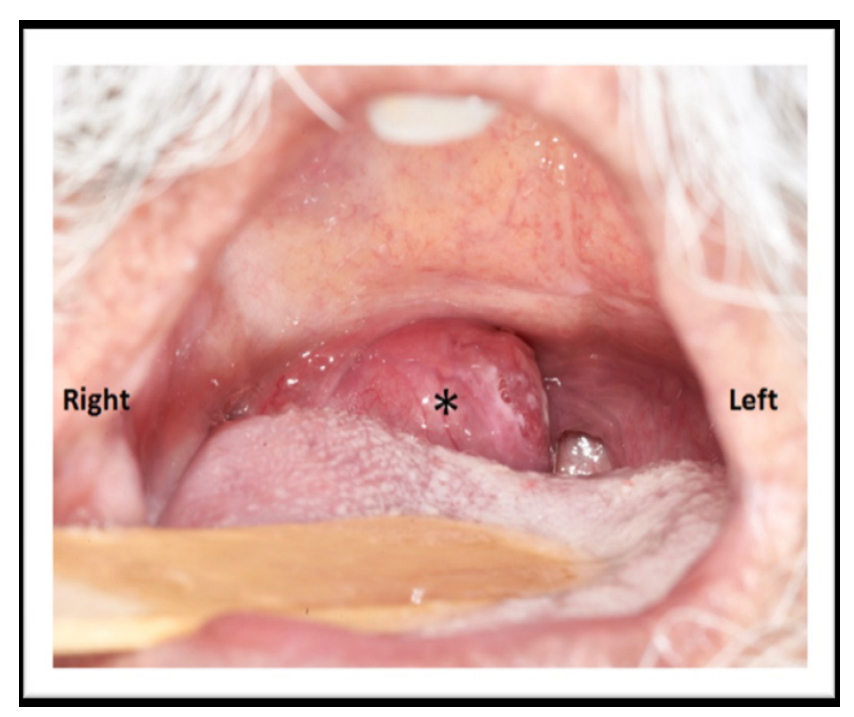

Figure I An example of unilateral swelling of the right tonsil - the eventual diagnosis in this patient was Lymphoma.

A recent study tried to evaluate whether isolated unilateral tonsillar enlargement was an indication for tonsillectomy. ${ }^{3}$ They reported that in patients presenting with unilateral tonsillar swelling alone (33) no malignancy was identified. From the group with unilateral tonsillar swelling with other associated symptoms (20) 9 malignancies were identified. They concluded that a 'watch and wait' policy would initially be more appropriate in patients with unilateral tonsillar swelling alone. They had relatively small study numbers so such a conclusion must be met with caution but it certainly highlights that unilateral tonsillar swelling alone may not be an indication for further investigations and that identifying associated symptoms is important. On the contrary, a study in 2004 identified 98 patients undergoing tonsillectomy for unilateral tonsillar swelling. ${ }^{4}$ Of these patients 23 malignancies were identified. They noted malignancy to be more common in patients over 45 , male, with the presence of lymphadenopathy and interestingly when the patient themselves had reported tonsillar enlargement. In our case, the patient remained concerned about malignancy and it was agreed to carry out tonsillectomy after mutual agreement between the patient and the surgeon.
Other clinicians have also struggled to reach a diagnosis when dealing with a painful, enlarging, exophytic tonsil lesion. ${ }^{5}$ Clinically, tonsil malignancy was suspected but the eventual diagnosis was a chronic, benign, inflammatory condition called Lichen sclerosis et atrophicus. ${ }^{5}$ So, tonsillectomy does certainly have a role in diagnosis of patients with unilateral tonsillar swelling but the presence of associated features seems to be a key in distinguishing high risk patients. In turn this certainly could be used to assess the timing of tonsillectomy in patients with unilateral tonsillar swelling alone. In our case, the reason for unilateral swelling of the tonsil was not cancer but without tonsillectomy it proved impossible to establish the benign diagnosis. It is now well established that unilateral tonsillar swelling is a risk factor for diagnosis of tonsillar malignancy. Along with this a complete, detailed examination and history is important to identify associated risk factors which in turn should prompt an urgent intervention. In the studies discussed the incidence of malignancy in the patients undergoing elective tonsillectomy for such reasons was small but however small they have to be regarded as significant. We conclude that tonsillectomy is associated with few post-operative complications and is a routinely performed operation. It seems that it is an appropriate investigation to undergo given the potentially devastating consequences of missing the diagnosis of tonsillar malignancy or delaying its treatment. Despite the low incidence of malignancy in tonsils, patients with asymmetric tonsil enlargement should be offered diagnostic tonsillectomy.

\section{Acknowledgments}

None.

\section{Conflicts of interest}

None.

\section{Funding}

None.

\section{References}

1. https://emedicine.medscape.com/article/848034-overview

2. Beatty MM, Funk GF, Karnell LH, et al. Risk Factors for malignancy in adult tonsils. Head Neck. 1998;20(5):399-403.

3. Sunkaraneni VS, Jones S E, Prasai A et al. Is unilateral tonsillar enlargement alone an indication for tonsillectomy? J Laryngol Otol. 2006;120(7):E21

4. Spinou C, Kubba H, Konstantinidis, I et al. Role of tonsillectomy in histology for adults with unilateral tonsillar enlargement. Resources. $\mathrm{Br}$ J Oral Maxillofac Surg. 2005;43(2):144-147.

5. Ajayi O, Stephens JC, Karim S, et al. Lichen sclerosis et atrophicus masquerading as tonsillar squamous cell carcinoma. J Laryngol Otol. 2009; 123(2):e10. 